\title{
Erratum
}

\section{Novel Synthesis of Naturally Occurring Pulvinones: A Heck Coupling, Transesterification, and Dieckmann Condensation Strategy}

David Bernier, Reinhard Brückner* Synthesis 2007, 2249.

In Table 4 , the heading of the $9^{\text {th }}$ column should be $\delta(\mathrm{C} 3)$ rather than $\delta(\mathrm{C} 1)$ and the heading of the last column $\delta\left(\mathrm{C} 1^{\prime}\right)$ rather than $\delta(\mathrm{C} 4)$. 